\title{
CAZÓN,
}

\section{PUEBLO DE EMPRENDEDORES}

\section{Gloria Edith Molinari}

Instituto de Investigaciones en Turismo.

Facultad de Ciencias Económicas -

Universidad Nacional de La Plata.

La Plata, Argentina.

(iD) 0000-0002-8193-4934

>11431472@econo.unlp.edu.ar
Elisabet Rossi

Instituto de Investigaciones en Turismo.

Facultad de Ciencias Económicas -

Universidad Nacional de La Plata.

La Plata, Argentina.

(iD) 0000-0001-9621-0827

Clisabet.rossi@econo.unlp.edu.ar

\section{RESUMEN}

El presente trabajo da cuenta de un Proyecto de Extensión de la Facultad de Ciencias Económicas (FCE) de la Universidad Nacional de la Plata (UNLP), durante los años 2018/2019 en la localidad de Cazón, distrito de Saladillo. Nuestra propuesta invita a pensar una gestión vinculada a la revaloración del patrimonio cultural, productivo y ambiental del destino que adecue el modelo turístico a las especificidades de cada territorio, y así evitar el consecuente deterioro de los valores endógenos de las comunidades locales. En este contexto, se abordó un turismo rural comunitario, donde la población local, sus productoras/es, artesanas/os y sus familias, ejercen un papel central en la organización de manera autogestiva, con la distribución equitativa de sus beneficios. El objetivo fue promover el desarrollo de pueblos rurales a través del turismo rural como articulador social de emprendimientos comunitarios, fomentando la asociatividad entre pequeños/as emprendedores/as. La metodología se desarrolló de manera participativa y horizontal, bajo la modalidad de taller, generando espacios de intercambio de experiencias y saberes que fortalecieron su desarrollo. A modo de conclusión, los procesos participativos, solidarios y colaborativos viabilizaron la organización de las y los pobladores para la toma de decisiones y fortalecieron el entramado social y productivo con mejoras en las condiciones de vida de la comunidad.

\section{PALABRAS CLAVE}

emprendedorismo $>$ patrimonio $>$ pueblos rurales $>$ turismo rural comunitario.

CAZÓN, PUEBLO DE EMPRENDEDORES

\section{Recibido}

22 de julio, 2021

Aprobado

30 de septiembre, 2021

Autoras:

Gloria Molinari - Elisabet Rossi
DOI

hitps://doi.org/10.24215/27186717e014
Ayana Revista de Investigación en Turismo

| Año 2 | Vol. 2 | № 01 Diciembre 2021 Mayo 2022

ISSN 2718-6717

Entidad editora Instituto de Investigaciones en Turismo, Facultad de Ciencias Económicas (Universidad Nacional de La Plata)

La Plata I Buenos Aires I Argentina 


\section{Ayana \\ Revista de Investigación

CAZÓN, TOWN OF ENTREPRENEURS

\section{ABSTRACT}

This paper reports on an Extension Project of the School of Economic Sciences (FCE) of the National University of La Plata (UNLP) during 2018-2019 in the town of Cazón, district of Saladillo.

Our proposal invites us to think about a management linked to the revaluation of the cultural, productive and environmental heritage of destinations, which adapts the tourism model to the specificities of each territory and, thus, avoids the consequent deterioration of the endogenous values of the local communities. In this context, rural community-based tourism was addressed, where the local population, its producers, artisans and their families exercise a central role in the organization in a self-managed way, with the equitable distribution of their benefits.

The objective was to promote the development of rural towns through rural tourism as a social articulator of community enterprises, encouraging associativity among small entrepreneurs. The methodology was developed in a participatory and horizontal manner, under the workshop modality, generating spaces for the exchange of experiences and knowledge that strengthened its development.

In conclusion, the participatory, supportive, and collaborative processes made it possible for the inhabitants to organize decision-making and strengthen the social and productive fabric by promoting improvements in the living conditions of the community.

\section{KEYWORDS}

entrepreneurship $>$ heritage $>$ rural villages $>$ rural community tourism.

\section{CAZÓN, CIDADE DOS EMPREENDEDORES RESUMO}

O presente trabalho conta sobre um Projeto de Extensão da FCE da UNLP, durante os anos 2018/2019 na cidade de Cazón, distrito de Saladillo.

Nossa proposta convida a pensar em uma gestão vinculada/ ligada à revalorização do patrimônio cultural, produtivo e ambiental dos destinos, que adapte o modelo turístico às especificidades de cada território e evite/ evitando, assim, a consequente deterioração dos valores endógenos das comunidades locais. Neste contexto, foi abordado um turismo comunitário rural, onde a população local, seus produtores, artesãos e suas famílias exercem um papel central na organização de forma autogerida , com a distribuição equitativa de seus benefícios.

O objetivo foi promover o desenvolvimento das aldeias rurais por meio do/através do turismo rural como articulador social dos empreendimentos comunitários, incentivando a associatividade entre os pequenos empreendedores. A metodologia foi desenvolvida de forma participativa e horizontal, sob a modalidade de workshop, gerando espaços para trocas/intercâmbio de experiências e conhecimentos que fortaleceram seu desenvolvimento. Em conclusão, os processos participativos, solidários e colaborativos possibilitaram que os habitantes organizassem a tomada de decisão e fortalecessem o tecido social e produtivo, promovendo melhorias nas condições de vida da comunidade.

\section{PALAVRAS CHAVE}

empreendedorismo $>$ herança $>$ aldeias rurais $>$ turismo comunitário rural. 


\section{Introducción}

En la actualidad, el turismo en espacios rurales se desarrolla con la participación cada vez mayor de sus comunidades, promoviendo otras formas de turismo que en algunos casos reproducen los modelos convencionales. Muchas de estas iniciativas se constituyen en escenarios con gran diversidad de recursos culturales y naturales que han sido considerados como una oportunidad. Tal es el caso del Turismo Rural Comunitario (TRC) que Kieffer (2018) plantea:

como un tipo de turismo de pequeño formato, establecido en zonas rurales y en donde la población local, a través de sus estructuras organizativas colectivas, ejerce un papel significativo en su control y gestión, ofreciendo actividades respetuosas con el medio natural, cultural y social, y con los valores de una comunidad, que permite disfrutar de un positivo intercambio de experiencias entre residentes y visitantes, donde la relación entre la y el turista y la comunidad es justa y los beneficios de la actividad son repartidos de forma equitativa (p. 9).

En este sentido, para que el ejercicio del TCR se convierta en una verdadera alternativa y no concluya en situaciones de despojo y de lucro privado, sin beneficios reales para las y los pobladores, se requiere del compromiso de todas/ os las y los actores institucionales, económicos, ambientales y sociales. Dicho compromiso es afianzado mediante la asociatividad, la cooperación y las prácticas solidarias permitiendo un desarrollo comunitario que satisfaga las necesidades y brinde oportunidades a las y los pobladores rurales (Vinasco, 2017).

Desde esta perspectiva, el trabajo territorial desarrollado durante el proyecto de extensión en la localidad de Cazón se enfocó en generar un sistema integrado cuyo eje estuviese en el desarrollo del turismo rural comunitario, donde se articulen diversos sub-ejes (cuidado del ambiente, patrimonio cultural, producción, emprendorismo, etc.) interconectados. De esta manera, se pusieron en común prácticas solidarias en el cuidado del ambiente; se documentaron historias de vida y experiencias, resguardando saberes y memorias como patrimonio del lugar, etc., lo que redunda en mejoras para cada una/o de las/os pobladores y grupos sociales en el aumento de la productividad de las prácticas viveriles y del emprendedorismo de actores comunitarios.

\section{Conceptos que atravesaron la práctica universitaria}

Desde la práctica universitaria desarrollada, partimos de una concepción de desarrollo comunitario integral en la que prime la valoración social de los lugares y la relación cara a cara entre las y los pobladores, y en el que la dimensión de lo local se constituya en un lugar privilegiado de articulación entre la racionalidad propia de los procesos de desarrollo y las identidades locales necesarias para dinamizar estos procesos (Manzanal, 2002). Desde esta concepción, Rodríguez (2010 como se citó en Gómez et al., 2016) plantea que:

el territorio es el depositario de un conjunto de significados y sentidos, que los distingue y diferencia de otros territorios. Es la expresión del patrimonio, del paisaje cultural, del espacio de solidaridad, del legado de la historia y lugar habitado por la memoria y la experiencia de las comunidades, es decir, es entendido como espacio construido por los grupos sociales a través del tiempo (p. 335).

Para complementar este concepto, cabe agregar que el territorio está integrado por numerosas/os actores sociales 
motivados por intereses diversos, siendo estos lugares arena de disputa entre instituciones, organizaciones (sociales, políticas y religiosas) que entran en convivencia con el Estado en sus distintas representaciones, sea municipal, provincial o nacional (Rossi et al., 2019).

Actualmente, el territorio rural - que históricamente se limitaba a la producción de alimentos - se diversificó en sus funciones y actividades hacia la multifuncionalidad: se entrelazan producción, conservación y cuidado de los recursos naturales, al tiempo que se articulan los múltiples servicios ambientales que dicho territorio ofrece para la regulación del clima, el abastecimiento de agua, de áreas protegidas, de centros culturales y recreación de los ciudadanos que brindan los servicios turísticos (Kieffer, 2018). Este proceso de diversificación se vincula con dos factores: una crisis sectorial que motiva a agricultores y ganaderos a buscar otras alternativas, y la visualización de una demanda creciente a partir de un sector dinámico, como es el turismo en tanto actividad social. La crisis ocurrida en Argentina en los años 90 promovió el desarrollo de emprendimientos turísticos desde las comunidades rurales y esto fue tomado como ejemplo en el resto de los países latinoamericanos.

La diversificación de la agricultura ha sido un patrón observado entre las y los productores de menor escala, sector que pierde competitividad en la producción de commodities. La modificación de la función productiva tradicional, entre las que se encuentra la incorporación de actividades no agrícolas y actividades turísticas, surge, en muchos casos, por la necesidad de diversificación del riesgo y de generación de ingresos adicionales a los agrícolas (Barrera 2006).

En este contexto, el TRC se instala como estrategia potenciadora de la multidimensionalidad rural territorial que amplia y diversifica las opciones productivas de las comunidades rurales al tiempo que complementa las economías de las familias rurales (Kieffer, 2018). Como consecuencia, se obtiene una serie de beneficios, tanto a nivel social como cultural, que favorecen a la población mediante alternativas colectivas como la autogestión y el asociativismo. Dichas modalidades no solo se manifiestan en un crecimiento económico material individual, sino también integran una dimensión sociocultural que mejora las condiciones de vida de la población local.

En sintonía con lo expresado, Barrera y Muñoz (2003) fundamentan la importancia del TRC señalando que:

fomenta la creación de empleo y del arraigo rural; el protagonismo de la mujer y las y los jóvenes; la revalorización del patrimonio cultural y ambiental; la creación de un mercado para la producción; el fomento del asociativismo; la mejora de la calidad de alimentos y artesanías; la multifuncionalidad de los espacios rurales y la pluriactividad de emprendimientos rurales (p. 18).

En párrafos anteriores se menciona el concepto de patrimonio, y su revalorización para la misma comunidad como atributo fundamental en los procesos de desarrollo turístico en el ámbito rural y transversal a la práctica universitaria. En este sentido, abordar el territorio productivo desde la perspectiva del patrimonio comprende el ejercicio de desnaturalizar y visibilizar las relaciones entre distintos sectores sociales y culturales, y respectivas sus prácticas. Asimismo, promueve el cuestionamiento y la repregunta acerca de dichas relaciones. Entendemos el patrimonio como el producto de una construcción social, en cuya configuración intervienen diferentes actroes sociales con el objetivo de satisfacer intereses específicos (Gómez et al., 2020).

Desde esta óptica, García Canclini (1999) se refiere al patrimonio como capital cultural, es decir, como "un proceso social que, como el capital, se acumula, se renueva, produce rendimientos y es apropiado en forma desigual por diversos 
sectores" (p. 6). Pensar el patrimonio evidencia tensiones sociales y culturales entre las comunidades asentadas en la zona, así como la naturalización del ambiente y de su utilización para fines productivos. Como plantea Gómez et al. (2016) "el patrimonio es un proceso dinámico que supone la selección, transformación de determinados elementos y su reconversión a partir de la creación de nuevos valores, por parte de diferentes grupos que están en tensión de intereses" (p. 336). Por lo planteado, el TRC se configura a partir de estos valores patrimoniales - tangibles e intangibles- para construir tramas socio-productivas sustentables en lo social-económico-ambiental basadas en relaciones sociales de respeto mutuo, autogestivas y pro-ambientales. Asimismo, el TRC es una oportunidad de empoderar a las comunidades, promover sus emprendimientos en torno a un turismo experiencial y mejorar los procesos de conservación de los recursos naturales. Para que dicha construcción de tramas socio-productivas se concrete y sostenga en el tiempo, es primordial la participación de todos los actores involucrados; en este sentido, Brett (1999) plantea que:

la participación implica un proceso de educación y capacitación en el cual las personas, asociadas y organizadas (conjunto de personas que persiguen un objetivo común) entre sí y con otros actores, identifican problemas y necesidades, movilizan recursos, y asumen responsabilidades para planificar, administrar, controlar y evaluar las acciones individuales y colectivas sobre las cuales ellos deciden (p. 4).

\section{El proyecto}

La comunidad de Cazón - conocida como la ciudad del millón de árboles - se encuentra ubicada en el partido de Saladillo, y cuenta con 209 habitantes, de acuerdo al último censo realizado en 2010. La población rural está en estado de decrecimiento como consecuencia de las escasas propuestas de inserción laboral, principalmente para las poblaciones de jóvenes y de adultos de la comunidad.

La mayoría de los que tienen trabajo, se dedican principalmente a las actividades viveriles, en forma privada o en el Vivero Municipal; otros se desempeñan en servicios de educación y salud.

El pueblo cuenta en su patrimonio con un repertorio de atractivos turísticos conservados durante más de cien años, casas cuidadas, jardines y huertas y calles arboladas, lo que demuestra que sus pobladores sostienen el compromiso de cuidar el lugar que habitan. Entre sus bienes patrimoniales, se encuentran la vieja estación del ferrocarril y las 210 hectáreas que forman parte del predio del Vivero Municipal. Este último, comprende distintos espacios no explotados y especies que conforman una reserva de flora, lugar dispuesto para el avistaje de aves. Asimismo, posee un espacio de recreación y esparcimiento conocido como Campamento de Cazón, el cual cuenta con edificio principal, baños, pileta, y zona de acampe. A unos metros de este se encuentran las caballerizas y el sector para la práctica de endurance. La atracción más significativa la constituye la Expo Vivero - ahora también la Expo Cactus- que recibe a miles de visitantes que recorren los puestos de artesanías, gastronomía, y asisten a distintos espectáculos artísticos y charlas sobre temáticas afines. El objetivo general del proyecto "Cazón, pueblo de emprendedores" fue promover el desarrollo de Cazón, a través del turismo rural comunitario en tanto articulador social de emprendimientos comunitarios. 


\section{Metodología}

La metodología se desarrolló de manera participativa y horizontal, bajo la modalidad de talleres que se realizaron cada 15 ○ 30 días, según la disposición de los asistentes. A los mismos asistieron un promedio de 13 emprendedores y 5 personas interesadas en iniciar un emprendimiento. Los talleres funcionaron como espacios de intercambios de experiencias y saberes que fortalecieron el desarrollo de la propuesta. La convocatoria fue realizada por el delegado municipal a las y los pobladores en general, a las y los emprendedores que venían realizando algunos proyectos productivos o servicios, y aquellos que quisieran incorporarse con alguna idea.

Durante la ejecución del proyecto, en el mes de septiembre de 2019, Cazón fue declarado Pueblo Turístico Bonaerense por la Secretaría de Turismo de la Provincia. Este programa del Estado provincial desarrolla prácticas de turismo comunitario que buscan generar arraigo, producción local e interés por la actividad turística en pueblos de menos de 2000 habitantes. Cabe aclarar que en el caso de Cazón, no se realizaron actividades previas y posteriores a su declaración, los pobladores fueron notificados al momento de la colocación de la cartelería correspondiente por las autoridades municipales y provinciales.

Los principales problemas detectados en los encuentros, manifestados por las y los presentes, fueron el desarraigo de la población juvenil, la pérdida de saberes de las y los pobladores antiguos, la escasa participación sostenida de vecinos atraídos por objetivos comunes, la falta de oportunidades laborales y la desarticulación entre instituciones. A ellos, se suman los problemas identificados por el equipo de trabajo, tales como la ausencia de redes comunitarias, observadas mediante las dificultades de las y los emprendedores del lugar para vincularse entre sí, ante la organización y funcionamiento de propuestas concretas.

Por otro lado, todos los eventos son organizados por las autoridades del municipio, donde la mayoría de los emprendimientos participantes no son locales y los pobladores locales no tienen poder de decisión en estos eventos. Dichas problemáticas generan en las y los pobladores una alta dependencia con las autoridades municipales en general, y en particular en la toma de decisiones, lo que dificulta el desarrollo de acciones comunitarias y autogestivas.

La participación en los talleres fue continua, en su mayoría mujeres jóvenes y adultas, que, si bien en algunos casos realizaban otras actividades, buscaban reforzar su fuente de ingresos.

\section{Resultados}

Mediante las reuniones, talleres, eventos territoriales y demás acciones de fortalecimiento de los emprendimientos se conformó una red social y solidaria que permitió la organización de las y los pobladores, despertando en ellos una cultura emprendedora. Es importante aclarar que a pesar de la escasa población de Cazón y del entusiasmo de los que participaron en los talleres, solo se sumaron aquellos emprendedores que tuvieron interés en el proyecto: un total de 20 de emprendedores, entre quienes ya venían trabajando en algún proyecto y aquellos que llegaron con una idea en mente; no logramos atraer al resto de las y los residentes.

A partir del proyecto, los participantes comenzaron a reunirse una vez cada quince días y a registrar lo charlado y consensuado en las reuniones. Dichos encuentros dieron como resultado el diseño y la posterior construcción de un 
corredor de emprendimientos, cuyo objetivo es visibilizar los saberes de los y las emprendedores/as y los procesos de producción que se desprende de ellos, como. Para tal fin, se diseñó un mapa con la ubicación de los emprendimientos y se instaló en la entrada del pueblo, acompañado de la cartelería correspondiente. Esto, permite al visitante acceder de manera autoguiada a cada emprendimiento: dulces caseros, artesanías con calabazas, tejidos, crochet, bicicletas recicladas, vivero, flores, jabones, alfajores, repostería, pinturas y esculturas en madera. Tanto el mapa como la cartelería fueron diseñados y ejecutados por dos artistas locales, con el acuerdo de todos/as. En este sentido y fortaleciendo la difusión del destino y los productos, las y los pobladores realizaron videos para mostrar lo que producen.

Se reformuló y diseñó el logo del destino que los/as identifica, con el consenso de todos en los talleres, se fortalecieron emprendimientos y crearon otros relacionados a lo productivo, artesanal y gastronómico. Para ello y junto al delegado Municipal se dictaron cursos de manipulación de alimentos, lo que permitió no solo diversificar la oferta gastronómica, sino utilizar de forma adecuada los productos locales, fundamentalmente las frutas del lugar.

En los talleres también surgió la necesidad, por parte de las y los emprendedores, de conocer qué tipo de estrategias de precios utilizar para comercializar sus producto. En ese sentido, se abordaron temas de costos a efectos de determinar un precio justo para sus productos. Uno de los participantes de los talleres, con conocimientos en las redes sociales, los asesoró acerca de la manera más ventajosa de presentar sus productos.

Paulatinamente, se comenzó a trabajar en forma asociativa, en la elaboración de distintas presentaciones de desayunos y almuerzos artesanales. En dicha confluyeron en la confección de bandejas, artesanas de la madera; en la de vasos, tazas y platos, artesanas de la cerámica; productoras de dulces, repostería y comida; y tejedoras al crochet, en la confección de los cubre tapas de los dulces. Durante el mes de abril 2021, se llevó a cabo la primera Feria de Mujeres Emprendedoras de Cazón.

\section{Conclusiones}

A partir de lo expresado, podemos concluir que los procesos participativos, solidarios y colaborativos viabilizaron la organización de las y los pobladores para la toma de decisiones y el fortalecimiento del entramado social y productivo. Asimismo, se promovieron mejoras en las condiciones de vida de la comunidad local y en el lugar que habitan. Por otro lado, también se visibilizaron los conflictos territoriales y las tensiones entre los diferentes actores, producto de la dependencia social, económica y cultural de las y los pobladores rurales con la ciudad cabecera, los cuales impactan sobre el desarrollo comunitario autogestivo, la toma de decisiones y la identidad del pueblo.

En este contexto, los procesos participativos, tal como menciona Breet (1999) permitieron abordar y reflexionar sobre dichas problemáticas y necesidades en la búsqueda de posibles soluciones, asumiendo responsabilidades en la organización y autogestión de los recorridos del corredor de emprendedores. Asimismo, la conformación de la red entre las y los emprendedores promovió el asociativismo, cooperación y prácticas solidarias, y brindó oportunidades a tanto a las y los pobladores rurales que la conforman como a aquellos que aún no forman parte de la misma.

Desde la perspectiva del TRC, las y los pobladores ejercen un papel significativo en su control y gestión, dado que generan espacios de intercambio de experiencias entre las y los visitantes e integrantes de la comunidad local (Kieffer, 
2018). Pensar esta alternativa de desarrollo turístico comunitario en Cazón requiere del compromiso de todas/os las y los actores sociales, institucionales, económicos y ambientales, en una relación respetuosa y justa, cuyos beneficios se distribuyan de manera equitativa.

\section{Referencias bibliográficas}

Barrera, E. \& Muñoz, R. (2003). Manual de turismo rural para micro, pequeños y medianos empresarios rurales. Serie de instrumentos técnicos para la microempresa rural. Fondo Internacional de Derechos Agrícola. Programa de Apoyo a la Microempresa Rural de América Latina y el Caribe (PROMER), pp. 13-18.

Barrera, E. (2006). Turismo rural. Nueva ruralidad y empleo rural no agrícola. Oficina Internacional del Trabajo, CINTERFOR.

Brett, Teddy (1999) Understanding organizations and institutions. In: Robinson, D. \& others (eds.) Managing Development: Understanding Inter-Organizational Relationships, 17-48. London: SAGE.

García Canclini, N. (1999). Los usos sociales del patrimonio cultural. En Aguilar Criado, E. et al. (Coords.). Patrimonio etnológico. Nuevas perspectivas de estudio, pp. 6-33. Sevilla: Consejería de la cultura. Instituto Andaluz del Patrimonio Histórico. Consejería de la cultura.

Gómez S, \& otros (2016). La construcción del patrimonio local como forma de intervención para descotidianizar el territorio. En 3ras Jornadas de Patrimonio y Desarrollo, pp. 330-340. La Plata: Icomos.

Gómez S. \& otros (2019). Aportes de la economía social y del turismo para el desarrollo comunitario del periurbano platense. Il Congreso Nacional de Economía Social y Solidaria. Universidad Nacional de Quilmes. Buenos Aires.

Gómez S. \& otros (2020). Reflexiones situadas sobre la extensión universitaria, la vinculación territorial y la integralidad. Trayectorias universitarias 6 (11).

Kieffer M. (2018). Conceptos claves para el estudio del Turismo Rural Comunitario. El Periplo Sustentable 34.

Manzanal M. (2002). Instituciones y gestión del desarrollo. Aportes para la construcción de una nueva Argentina. Realidad económica 118, 51-67.

Vinasco Guzmán, M. C. (2017). Marco teórico para la construcción de una propuesta de turismo rural comunitario. Revista de Investigación Agraria y Ambiental 8(1). 